\title{
Las tecnologías de la información desde el punto de vista de género: posturas y propuestas desde el feminismo
}

\author{
Verónica SAnZ GonZÁleZ* \\ Instituto de Filosofía, CSIC
}

RESUMEN. El enorme desarrollo que en las últimas décadas han experimentado las tecnologías de la computación y las comunicaciones ha provocado diversos debates teóricos y dilemas éticos entre los que la perspectiva de género ha permanecido tradicionalmente ausente. Algunos análisis feministas sobre tecnologías digitales han surgido dentro de la corriente CTS, siendo mucho más escasos en el ámbito de la ética computacional En este trabajo expondremos la historia de la perspectiva de género en los estudios sobre tecnologías computacionales, y mostraremos la pertinencia y la importancia de esta perspectiva para una adecuada comprensión del fenómeno, tanto desde el punto de vista de la relación entre tecnología y sociedad, como de los problemas éticos de los que se ocupa la ética computacional.

Palabras clave: TICs, género, feminismo, tecnología, constructivismo social, ética computacional

\section{Introducción}

En los últimos quince años, concretamente desde principios de los 90, se ha producido una productiva intersección entre los denominados estudios de Ciencia, Tecnología y Sociedad (CTS) y los estudios
ABSTRACT. The enormous development that computer and communication technologies have experienced during last decades has caused several ethical problems and theoretical debates. However, a gender perspective has not been traditionally included in these researches. Some of the first feminist analyses about digital technologies have emerged within STS, being much scarce in the discipline of Computer Ethics. In this paper I will briefly expose some of these analysis, and will argue for the importance of the gender perspective for an appropriate understanding of this phenomenon, both regarding technology and society relation and in relation to the ethical problems which are the concern of Computer Ethics.

Key words: ICTs, Gender, Feminism, Social Constructivism, Computer Ethics.

feministas sobre tecnología o de Ciencia, Tecnología y Género (CTG), al incorporar estos últimos la postura sociológica constructivista en el análisis de las tecnologías. Esto significa que comparten la perspectiva de entender las tecnologías como resultado tanto de procesos tecnológicos como 
sociales (son, por tanto, un producto sociotécnico). En oposición a las teorías sociológicas clásicas (que reducían su estudio a la estructura externa y a las relaciones sociales, y, en lo referente al tecnología, al análisis del impacto de éstas en la sociedad), las nuevas corrientes en CTS consideran que el desarrollo de los artefactos tecnológicos (y las teorías científicas) se construyen conjuntamente con la aparición de nuevos tipos de relaciones sociales.

La mayoría de estas autoras ${ }^{1}$ asumen como propios conceptos desarrollados por autores clásicos en CTS tales como «sistema sociotécnico» $\left(\right.$ Hughes $\left.^{2}\right)$, «flexibilidad interpretativa de los artefactos»y «clausura y estabilización» (Pinch y Bji$\operatorname{ker}^{3}$ ), «red heterogénea de actores humanos y no humanos» $\mathrm{y}$ «traslación y delegación a los actantes no humanos» (Latour ${ }^{4}$ ). No obstante, como ya hemos apuntado, critican a las corrientes dominantes (concretamente al programa SCOT -Social Construction of Technology5- y a la Teoría del Actor-Red) su silencio acerca de cómo las estructuras perdurables de poder subyacentes en la sociedad (como por ejemplo el sistema patriarcal de dominación masculina) pueden influir en los desarrollos tecnológicos. Este tipo de estructuras de poder, que se muestran perdurables a lo largo del tiempo, producen situaciones discriminatorias que no permiten procesos de renegociación entre los diferentes actores en situación de igualdad.

La aportación de las feministas constructivistas a estas teorías sociológicas es la de incluir la categoría de género como una variable explícita de análisis en la co-construcción de tecnología y sociedad. Así, consideran que también se produce un proceso de co-construcción entre el género y la tecnología. La tecnología no sólo «afecta» a las relaciones y a la definición de género, sino que, afirman, en toda innovación tecnológica se produce una renegociación de las relaciones y una articulación de las identidades de género que van a ser performadas 6 con el uso de ese artefacto. Por tanto, también las fases de uso y consumo (que es donde encontramos más mujeres) deben ser analizadas como parte constitutiva del desarrollo de las tecnologías. Autores como Grint y Gill ${ }^{7}$ ven en el hecho de que los estudios CTS obvien la categoría de género una actitud clásicamente sexista criticada por toda la tradición de epistemología feminista (que ha demostrado que la relevancia del género no emerge hasta que se usa como herramienta de análisis). La neutralidad de los actores respecto al género es una ilusión, dice Haraway ${ }^{8}$, ya que todos somos seres socialmente sexuados y generizados, y esa parte de nuestra identidad subjetiva participa en la ideología y las acciones que efectúan los actores dentro de las redes tecnocientíficas.

Judy Wajcman denomina esta productiva intersección entre feminismo y constructivismo «Tecnofeminismo», y la caracteriza como «la corriente de CTS que subraya la contingencia y la heterogeneidad del cambio tecnológico, que se localiza a su vez dentro de redes sociales más amplias, pero introduciendo un espacio para la agencia de las mujeres dentro de los cambios tecnológicos» 9 .

\section{Ciberfeminismo}

$\mathrm{Si}$ bien las feministas constructivistas que antes hemos apuntado se sitúan, a pesar de las críticas, dentro de la corriente CTS, en el caso específico de las tecnologías de la información y las comunicaciones surgió en los años 90 un nuevo enfoque que ha tenido gran acogida entre el feminismo, siempre abierto a nuevas esperanzas políticas. 
Una parte importante de esta corriente, denominada Ciberfeminsmo, proviene de la tradición del feminismo cultural de corte postmoderno. Una de las características de este tipo de feminismo es que, como su nombre indica, limita su análisis a las tecnologías digitales o tecnologías de la información y las comunicaciones (conocidas por las siglas «TICs»), dejando de lado otro tipo de tecnologías tradicionalmente objeto de estudio del feminismo (como las tecnologías domésticas o las tecnologías reproductivas). El término Ciberfeminis$m o$ fue acuñado por el grupo australiano VNS Matrix en su manifiesto de 1991, en el que se proponen explorar las identidades y sexualidades en el ciberespacio. Todas ellas provienen de una nueva generación de mujeres jóvenes que ya han crecido utilizando las tecnologías digitales, y que en su mayor parte pertenecen a culturas populares de los años 90 como el ciber-punk o las ciber-girrls (ciberguerrilleras). El Ciberfeminsmo no deja de reconocer el sesgo patriarcal en el diseño y la producción industrial de las tecnologías (donde las mujeres conforman la mayoría de la mano de obra menos cualificada y donde la discriminación para llegar a los puestos más altos es todavía muy patente). Sin embargo, estas autoras insisten en que las nuevas tecnologías de la información han creado un nuevo espacio caracterizado por ser abierto, difuso y no jerárquico (incluso aunque estas características hayan podido surgir como un factor emergente no del todo predecible por sus primeros diseñadores) que se ajusta sorprendentemente bien a lo que ellas consideran «caracteres femeninos».

Donna Haraway acuñó por primera vez para el feminismo el término de «ciborg» en su clásico ensayo Manifiesto Cyborg, publicado en $1985^{10}$. La influencia de este concepto en los debates feministas de los años 90 ha sido importantísima, además de que ha ayudado a extender la crítica feminista a lugares donde no solía ser escuchada. El término «ciborg» es definido por Haraway como «un organismo cibernético, un híbrido de la máquina y el organismo». La idea principal del concepto de ciborg es que difumina las fronteras entre las categorías de «natural»y «artificial», por lo que, a su vez, también lo hace con las de varón y hembra al difuminarse las diferencias biológicas entre los seres humanos. Así, las mujeres serán liberadas de un destino tradicionalmente marcado por su biología, y se les abre las puertas a un mundo más igualitario y plural, donde la libertad es más plausible y las posibilidades del feminismo mucho más diversas.

El famoso texto de Sadie Plant Ceros y Unos ${ }^{11}$ analiza la idea de que las tecnologías digitales producen sistemas y procesos tecnológicos fluidos donde las cualidades más valoradas son las de la comunicación y la cooperación, lo que, para Plant, constituye «el modo distintivo de ser femenino». La supuesta habilidad femenina de realizar diferentes tareas al mismo tiempo, por ejemplo, es una de las características de las nuevas tecnologías, que parecen adaptarse cómodamente a esa «identidad femenina» ${ }^{12}$ que en el pasado era valorada negativamente. Sherry Tukle ${ }^{13}$, por su parte, insiste en la idea de que Internet ofrece la posibilidad de experimentar creativamente con los múltiples aspectos de la propia subjetividad (jugar a ser otro/a) que pasan a ser nuestras identidades virtuales. Para Turkle, Internet es «la expresión material de la filosofía postmoderna», pues desafía la concepción moderna de una identidad única poniendo en su lugar subjetividades múltiples y fluidas.

Las posturas de Plant y Turkle suponen, no obstante, cierto tipo de esencialismo tanto acerca de la feminidad como 
de las tecnologías digitales. A pesar de su presentación anarco-activista, de sus ideas se deduce cierto inmovilismo político ya que, si consideran las tecnologías digitales como inherentemente femeninas y liberadoras, no parecen ser necesarias ulteriores acciones políticas feministas. En este sentido Haraway se desmarca de posturas esencialistas como la de Plant porque, en última instancia, acaban refiriendo a los cuerpos femeninos manteniendo el concepto tradicional de una esencia marcada irremediablemente, fija e inmutable, cuya visibilidad (aunque pretenda ser reivindicada como ventaja) ha sido el fundamento de la exclusión de esas identidades por el sistema de poder ${ }^{14}$.

En opinión de Wacjman las tres autoras mencionadas asumen una concepción utópica de las nuevas tecnologías digitales fundada en la aparente facilidad en el nuevo espacio de deconstruir las identidades tradicionales y crear otras alternativas. Lo que no explica la concepción utópica es el porqué de la persistente situación de inferioridad de las mujeres en el ámbito tecnológico. Este optimismo, apunta Wacjman, ha de ser entendido como una reacción al pesimismo de los estudios feministas sobre tecnología de los años $80^{15}$.

El optimismo de algunas ciberfeministas coincide en ese punto con cierto tipo de literatura bastante extendida acerca de los efectos de las tecnologías digitales y la creación de la nueva «Sociedad de la Información». Uno de los mayores exponentes de esta postura es el mundialmente conocido Manuel Castells ${ }^{16}$, para quien la nueva «red de redes» ha revolucionado no sólo la tecnología sino también la sociedad, que gracias a las nuevas tecnologías podrá solventar los problemas de desintegración e individualismo que la amenazan desde hace unas décadas. Este tipo de tecnologías, según Castells, permiten nuevas formas de conexión y socialización entre los individuos, dando lugar a comunidades virtuales ${ }^{17}$.

Esta visión optimista tanto del Ciberfeminismo como de los defensores de la nueva «Sociedad del Conocimiento» no se ha librado de múltiples críticas desde otros sectores del feminismo. Algunas de estas críticas son:

a) A pesar de las posibilidades que las TICs ofrecen para crear «comunidades virtuales», las comunidades reales (materiales) siguen siendo muy importantes, existiendo en ellas diferencias materiales y de poder, según la clase social, el país o región de origen, la raza y, por supuesto, el género. Estas diferencias se refieren usualmente con el término «brecha digital».

b) En algunos casos, esta situación de desigualdad no sólo no se resuelve gracias a Internet, sino que incluso se magnifica. En muchos casos se traslada a la red los valores, identidades y estereotipos discriminatorios de la sociedad en general, y gracias al enorme poder de transmisión de estas tecnologías el resultado es un mayor grado de discriminación (como ocurre por ejemplo con la enorme expansión de la pornografía en Internet).

c) La «cultura hacker» que es mitificada en estas posturas, representa un tipo de cultura masculina, blanca y de clase media, como ponen de manifiesto muchas feministas. No es por tanto tan cierto que sea esta la pretendida «cultura de la libertad».

d) La escasa participación de las mujeres en la sociedad de la información tanto en las fases de diseño de las tecnologías como en la producción de contenidos no es tenida en cuenta, ni son analizadas debidamente las consecuencias que esto tiene para las 
características que definen a la actual Internet.

\section{2. Ética computacional}

$\mathrm{Si}$, como hemos apuntado en la introducción, los estudios sociales de la tecnología han tardado dos décadas en incluir de algún modo el análisis de género, otras disciplinas como la Ética Computacional, con un origen posterior, tienen los mismos tipos de carencias. El término Ética Computacional es una de las múltiples traducciones del inglés Computer Ethics, el nombre inicial que tomó esta disciplina en los años 80. Es español, no obstante, hay otras posibles traducciones como Ética Informática, y las más modernas Ética Informacional, Ciberética o Infoética, que ya refieren no sólo a las tecnologías de la computación como sugieren las primeras denominaciones, sino también a las tecnologías de las comunicaciones que las primeras han propiciado $^{18}$.

Debido a que las ciencias de la computación y la ingeniería informática son unas de las disciplinas tecnocientíficas más modernas, el nacimiento de la ética computacional es aún más tardía, apareciendo primeros textos hacia el año 1985. La justificación de esta nueva corriente se encuentra en la necesidad de solucionar ciertos problemas morales que provocan el uso de los ordenadores y las nuevas redes de comunicación. Desde el nacimiento de la ética computacional se viene produciendo un debate interno que podemos denominar como el de la especificidad de los dilemas éticos del ámbito digital. Desde algunas posturas, los problemas morales planteados no son más que variantes de viejos problemas éticos que encontramos en otros ámbitos de la vida, por lo que la ética computacional no sería sino una «ética aplica$d a »$ donde aplicar los conceptos de las éticas tradicionales sin necesidad de ulterior desarrollo teórico ${ }^{19}$. Otros autores, por el contrario, defienden que las TICs han provocado nuevos y particulares problemas no conocidos anteriormente que requieren de un análsisis específico ${ }^{20}$.

Dentro del amplio espectro que actualmente puede considerarse parte de la ética computacional, podemos definir, no obstante, varias corrientes diferenciadas por los ámbitos de los que surgieron y por sus diferentes clasificaciones de los asuntos que consideran:

a) Orientación Académica: Esta corriente se localiza en un punto intermedio entre la filosofía académica y los estudios de ciencias de la computación e ingeniería informática en las universidades. Desde hace aproximadamente una década podemos encontrar en los estudios de ingeniería informática y similares de las principales universidades del ámbito anglosajón materias con el nombre de «ética computacional», así como libros de texto, revistas especializadas $^{21}$, congresos, etc. Esta disciplina es considerada en el ámbito académico ingenieril una ética aplicada, en cuyos casos prácticos se pueden aplicar las teorías éticas tradicionales como el utilitarismo, las éticas deontológicas o la ética de la virtud de corte aristotélico ${ }^{22}$.

b) Orientación Profesional-Empresarial: Una gran parte del desarrollo de la ética computacional está ligada al tema del surgimiento ( $\mathrm{y}$ necesidad de afianzar) la informática como una actividad profesional independiente. Un elemento importante de todo grupo profesional consiste en desarrollar un código deontológico específico, que en este caso ha propiciado un debate sobre si los profesionales de la informática deben considerarse una 
rama de la ingeniería o no. En realidad muchos de los profesionales que trabajan en informática no poseen el título de ingenieros. No obstante, en la mayoría de los países las principales asociaciones profesionales han elaborado o elaboraron sus códigos a imagen de otras asociaciones ingenieriles. Como tales guías prácticas, esta corriente carece de desarrollo teórico, y sus posturas están más cerca de análisis utilitaristas de costes y beneficios. Ejemplos de este tipo de códigos son los de la Association for Computing Machinery (ACM) o el Institute of Electrical and Electronics Engineers (IEEE) en los Estados Unidos, o la British Computer Society en el Reino Unido. En general, los asuntos comúnmente referidos como propios de la ética computacional tal y como aparecen por ejemplo en la Stanford Enciclopedia of Philosophy ${ }^{23}$ son los siguientes: privacidad, propiedad intelectual (tanto de software como de contenidos en la red), los crímenes o delitos cibernéticos, la responsabilidad profesional, la salud y la seguridad en los lugares de trabajo, y los problemas relacionados con la globalización en Internet. Algunos códigos como el de la ACM incluyen también asuntos de justicia social como el acceso a las TICs (lo que se conoce como el problema de la «brecha digital»).

c) Orientación Cibercultural (o Ciberlibertaria): Al mismo tiempo que las anteriores perspectivas, los escritos más populares sobre ciberética (en el sentido más amplio de la ética computacional) provienen de los propios usuarios de los ordenadores y las redes, que se han organizado a través de Internet para reivindicar sus derechos. Este movimiento se llama también «cibercultura» y pertenecen a él normalmente usuarios entusiastas con elevados conocimientos de informática (generalmente varones jóvenes y blancos). Sus posturas están relacionadas a menudo con movimientos como el llamado «ciberpunk» o la «cultura hacker», y también con cierta literatura de ciencia ficción acerca del futuro de la especie humana en un mundo dominado por los ordenadores. Los dilemas éticos que más les preocupan tienen que ver con la libertad en la red, tanto de expresión como de libre acceso y libre comunicación de la información ${ }^{24}$.

Debido a la larga tradición de escasa comunicación entre distintas disciplinas académicas (a pesar de las constantes llamadas a la interdisciplinaridad en los últimos años), la ética computacional tanto en su vertiente más teórica como en la profesional, no ha incluido en sus análisis las críticas largamente desarrolladas por la corriente CTS, y aún menos, las de los estudios feministas sobre tecnología. Por ello encontramos que la mayoría de acercamientos a la ética computacional adolecen de asunciones tácitas que han sido puestas al descubierto por los principales autores de los estudios sociales de la tecnología. Como señala Alison Adam, en general estas tres corrientes suscriben de manera tácita lo que en filosofía de la tecnología se conoce como determinismo tecnológico y tecnooptimismo. El primero consiste en la creencia en la inevitabilidad de los desarrollos tecnológicos y la idea de que afectan a la sociedad pero ésta no influye en sus desarrollos. Esta concepción tiene como consecuencia la ausencia de análisis y crítica al modo en que se producen los artefactos tecnológicos. El determinismo tecnológico en general 
potencia una postura más bien inmovilista en cuanto a lo dilemas éticos y políticos en el ámbito de la tecnología, lo que desde CTS se relaciona con posturas políticas conservadoras de corte liberal. Asimismo podemos observar cómo todas las corrientes de la ética computacional profesan un manifiesto tecnooptimismo o tecno-entusiasmo (idea de que los desarrollos tecnológicos, en general, conducen inevitablemente al progreso social), considerando además que la revolución de las TICs supone el cambio tecnológico más revolucionario en la historia humana. Desde este punto de vista, la ética computacional sólo debería ocuparse de algunos usos malintencionados de estas tecnologías. Esta postura optimista y acrítica fundada en el determinismo tecnológico y las bondades del liberalismo rechaza en general cualquier intromisión institucional en lo que pretenden sea «una sociedad libre de regulaciones». En el caso concreto las desigualdades sociales la postura general es que las tecnologías digitales producirán por sí mismas la igualdad y la democracia, sin necesidad de cambios en la estructura profunda de la sociedad.

\section{La perspectiva de género en los estudios de ética computacional}

La teoría crítica feminista, por su parte, tampoco se ha librado del problema de la escasa comunicación entre disciplinas, incluso dentro del propio feminismo. Como veremos a continuación, las escasas y muy recientes incursiones feministas en el campo de la ética computacional provienen generalmente del feminismo liberal y de la ética feminista, pero no incluyen los importantes desarrollos teóricos que desde los estudios feministas en ciencia y tecnología se han conseguido en los últimos años. Estos han realizado una importante labor al analizar la asociación histórica entre masculinidad y tecnología a un nivel muy profundo, lo que podría ser utilizado por la ética y la política feministas como base para propuestas de acción que obtengan mejores resultados que los que el feminismo liberal ha conseguido hasta ahora en lo que respecta a la escasa participación de las mujeres en los ámbitos científico-tecnológicos. Algunos trabajos recientes como el de Alison Adam ${ }^{25}$ intentan, precisamente, acercar ambos tipos de análisis con el fin de obtener una teoría y una práctica feministas más eficientes para acercarnos al objetivo de la igualdad entre los sexos en el ámbito de las TICs.

A pesar de su escasa repercusión en las disciplinas más clásicas e influyentes del ámbito académico, el feminismo posee una larga tradición de pensamiento que ha dado como resultado diferentes teorías y propuestas ético-políticas. Por eso, Adam en su último trabajo muestra la necesidad de incluir los análisis de género en la disciplina de la ética computacional (o informacional). Adam está convencida de que muchos de los problemas morales de la ética computacional tal como la hemos descrito en la sección anterior no pueden resolverse, ni tan siquiera comprenderse en su total dimensión, si no se analizan en términos de género, lo que supone, a efectos prácticos, utilizar las herramientas teóricas y prácticas desarrolladas por el feminismo en las últimas décadas.

A pesar de esta tradicional carencia de análisis de género en la mayoría de las disciplinas y, como hemos apuntado, también en el campo de la ética computacional, en los últimos años ha comenzado a aparecer cierta literatura acerca de temas de género que empieza a incluirse como parte de las investigaciones, textos y conferencias de ética computacional, dentro de lo que en ese campo se denomina «asuntos de justicia social y acceso». 
El tratamiento al problema de género en la ética computacional ha tenido hasta el momento dos tipos de acercamientos:

a) Por un lado se han elaborado numerosos análisis cuantitativos sobre el número de mujeres en los distintos rangos de los sectores de las TICs. Este tipo de estudios, normalmente realizados en las universidades, se centran en el persistente escaso número de mujeres en las tecnologías computacionales y los problemas o barreras para su acceso (tanto en el caso de los estudios universitarios como en el empleo relacionado con las TICs ${ }^{26}$. Debido a que es el paradigma actualmente preeminente en las ciencias sociales y en el mundo empresarial, los estudios cuantitativos son mucho más numerosos $\mathrm{y}$ mejor valorados a la hora de ser publicados o financiados como proyectos.

b) Por otro lado, muy recientemente se han comenzado a realizar algunas investigaciones acerca de las supuestas diferencias entre las actitudes éticas de hombres y mujeres en la toma de decisiones sobre problemas concretos que provocan las nuevas tecnologías. Estos estudios, a menudo relacionados con la ética empresarial y profesional, suelen consistir en cuestionarios realizados a usuarios/as y trabajadores/as que usan estas tecnologías proponiéndoles casos concretos de dilemas éticos en los que tendrían que tomar una decisión, con la intención de detectar si existen diferencias relevantes entre hombres y mujeres 27 .

El primer tipo de estudios sobre el número de mujeres se enmarca dentro de lo que comúnmente se denomina feminismo liberal. No hay duda de que es un problema muy serio la escasez de mujeres en el sector, y que es necesaria la constante actualización de los datos para hacernos cargo de la situación real ${ }^{28}$. No obstante, la mayoría de estudios cuantitativos de este tipo carece de una reflexión más profunda acerca de las causas de este fenómeno, ofreciendo como propuestas medidas políticas de igualdad formal (en la educación, el acceso al empleo, etc) que han mostrado no ser demasiado efectivas en las últimas tres décadas. Una excepción a este tipo de estudios son las investigaciones de Cecilia Castaño sobre la situación en España, cuyo extenso análisis de la situación de las mujeres en los distintos sectores de las TICs (en el empleo, en el sistema educativo y en el uso de Internet) se complementa con un considerable recorrido por los desarrollo teóricos feministas acerca de la relación entre género y TICs, y con un conjunto de propuestas consecuentes con las conclusiones de su estudio ${ }^{29}$.

El segundo tipo de estudios, tal y como son presentados por Alison Adam, suponen un intento de incluir una perspectiva cualitativa no recogida por los estudios estadísticos. Sin embargo, esta perspectiva corre el riesgo de caer en un esencialismo femenino (lo que de hecho ocurre a menudo) al carecer de una teoría de género suficientemente desarrollada y desconocer los debates internos de la ética feminista desde los años 70. Estudios como el de Mason y Mudrack ${ }^{30}$ o Kreie y Cronan ${ }^{31}$ concluyen que las mujeres son «más éticas» que los hombres (en el sentido de que prestarían más atención a los problemas morales en sus respuestas, y que entre sus preocupaciones aparecen más a menudo los asuntos de responsabilidad social y medioambiental). A pesar de ello, estos autores no incluyen ninguna referencia a la tradición feminista de la ética del cuidado, 
por ejemplo, ni se cuestionan si esas conclusiones pueden deberse a los estereotipos culturales de género en los que las mujeres son socializadas y educadas desde la infancia. Por otro lado, la metodología utilizada en estos estudios es también muy cuestionable. En lo relativo a los sujetos a los que se les realizan los cuestionarios, no siempre están representados todos los grupos o sectores, extrayéndose conclusiones generales sólo con las respuestas de una parte. Por otro lado, las opciones de respuesta en dichos cuestionarios son, o bien respuestas de SÍ o NO, o bien de escalas tipo Likert ${ }^{32}$ sin posibilidad de más términos intermedios, a lo que se añade la alta probabilidad de inducción de las respuestas por las preguntas (los encuestados responderían lo que supone que es «éticamente correcto» y no lo que quizá harían en una situación real). Esto inevitablemente crea un sesgo en los resultados, como han criticado muchas autoras feministas, que no representa lo que ocurre en situaciones reales. Para poder considerar medianamente adecuados los acercamientos cualitativos, Adam sugiere la necesidad de realizar estudios etnográficos en los ámbitos de trabajo (que proporcionarían una visión más realista de la situación), así como incluir la perspectiva de género en los estudios sobre gestión empresarial.

Para ejemplificar como podría aplicarse la perspectiva de género en la ética computacional, Alison Adam propone en los tres últimos capítulos de su libro el tratamiento de casos prácticos que, aun formando parte de los dilemas éticos tradicionalmente tratados por la ética computacional, no incluyen las cuestiones de género como parte de ese análisis. Esto es muy patente incluso en ciertos casos en los que la cuestión del género aparece de forma muy visible. A continuación vamos a exponer algunos de estos casos para mostrar cómo incorporando la perspectiva de género estos problemas se nos presentan de una manera más completa:

\section{Caso 1) Intimidación y Ciber-acoso en Internet}

El asunto de los ciber-delitos es uno de los que más atención ha recibido tanto desde la orientación ética como en los aspectos legales y de intervención policial. Especialmente relevante es el caso de la pornografía infantil y las redes de pedofilia en Internet. El factor más importante desde la perspectiva de género, que no es suficientemente puesto de manifiesto como punto de partida en los debates generales, es el hecho de que la mayoría de las víctimas de estos crímenes son mujeres o niños/as. Podríamos decir que el ciberacoso es, en realidad, una forma de acoso «generizada en femenino». No hay duda de que estas actividades ilegales suponen un tipo de violencia que se ejerce a través de los medios telemáticos, o bien utilizando éstos para perpetrar en última instancia actos de violencia física en el sentido tradicional del término. En lo que respecta al debate ético y legal de este tipo de delitos, se presenta el tradicional dilema en ética informacional de si la aparición de estas nuevas tecnologías suponen un cambio cualitativo en las características de los delitos cibernéticos, o bien no son más que reproducciones en el ciberespacio de la violencia sexual y el abuso a mujeres y menores en el mundo «real». En los últimos años se ha observado un considerable aumento del intercambio de imágenes sin consentimiento y de actividades relacionadas con la pornografía en Internet, debido a las posibilidades de anonimato y de conexión entre sujetos con las mismas «preferencias» que ofrece la red. Lo que parece evidente en todo caso es que el ciberespacio reproduce en 
gran medida los estereotipos culturales de género, como hemos argumentado anteriormente. A pesar de la creencia tácita de los tecnooptimistas en el carácter intrínsecamente liberador y democrático de Internet, el estudio de casos concretos de ciber-delitos muestra una realidad muy diferente. Por ello creemos que sería de gran utilidad integrar en el estudio de estos ciber-delitos las investigaciones que la teoría feminista (especialmente en el ámbito del derecho) ha venido desarrollando acerca de la violencia sobre las mujeres, el acoso sexual, la discriminación por cuestiones de género, etc. En este sentido nos parece importante resaltar cómo el acoso o el intercambio de pornografía femenina o infantil en Internet refieren en última instancia al control sobre los cuerpos (femeninos o infantiles). Esto es un problema que tradicionalmente ha ejercido el género masculino sobre los cuerpos de seres que consideran «objetos de deseo» y susceptibles de ser «violados» (física o metafóricamente). En el fondo de esta cuestión se encuentra la diferente posición de poder entre los géneros, y la situación de inferioridad de uno respecto al otro, lo que es el tema principal de estudio de la teoría feminista ${ }^{33}$.

\section{Caso 2) Privacidad en Internet}

El problema de la privacidad en Internet fue unos de los primeros asuntos tratados por la ética computacional. Dentro de este ámbito se incluyen problemas como el de la privacidad de las bases de datos que incluyen información personal de los ciudadanos tales como su dirección, sus datos fiscales, sus antecedentes penales o su historial médico. Otro de los problemas que se plantean es la obtención de las direcciones de correo electrónico o el historial de las búsquedas de los usuarios de Internet para elaborar «perfiles de consumidor» que servirían para bombardear de publicidad sus buzones de correo. Sin embargo otro tipo de ataques a la intimidad no son considerados con la misma intensidad en esta literatura. Así ocurre en el caso anterior del ciber-acoso o la pornografía en Internet. En estos ciber-delitos se utiliza de forma no consentida el correo personal de los usuarios, a la vez que se distribuyen imágenes personales no autorizadas. Estos problemas, como hemos afirmado antes, no afectan del mismo modo a hombres y a mujeres. El uso de imágenes sin consentimiento de las afectadas constituye una de las amenazas más importantes a la privacidad de las mujeres (y los menores) en Internet. A primera vista puede no parecer obvio el hecho de que la privacidad «informacional» (en la red) esté relacionada con la privacidad física (o corporal). La relación se produce de modo indirecto pero no por ello sus efectos son menores, pues es muchos casos esa barrera entre el ciber-acosador (que se introduce en los chats con identidades falsas o envía correos electrónicos) y la persecución real se rompe fácilmente, y la víctima es atacada de una u otra manera. Los ataques a la privacidad «informacional», además de posibilitar ataques a la integridad física de las mujeres amenazan también la «privacidad en la toma de decisiones» de éstas, en tanto que limitan sus capacidad de decidir autónomamente sobre el uso de la red para su vida privada o profesional (por ejemplo decidir si comenzar un negocio a través de Internet o concertar una cita a través de un chat $^{34}$ ).

¿Cómo podemos abordar este tema utilizando la perspectiva de género? En primer lugar podemos comprobar cómo la teoría feminista ha tratado extensamente el concepto de privacidad, si bien desde un punto de vista general ${ }^{35}$. En este sentido han puesto de manifiesto la 
contingencia histórico cultural de este concepto a lo largo de los dos siglos pasados, y han analizado la relación de este concepto con la dicotomía tradicional entre público/privado, entre vida pública (los ámbitos político y económico en principio exclusivamente masculinos) y vida privada (relativo al ámbito doméstico y exclusivo de las mujeres). Algunas autoras como Kramer y Kramarae $^{36}$ resaltan que debido a que tradicionalmente las mujeres han carecido del derecho a la privacidad (empezando por no poder poseer propiedad privada) y a la autonomía en las decisiones sobre la propia vida, es mucho más difícil saber cuándo la privacidad de las mujeres está siendo violada. Los asuntos sobre privacidad que trata la ética computacional refieren normalmente al ámbito de «lo público» (datos legales, fiscales, etc), mientras que los temas relacionados con la privacidad sexual y del cuerpo de las mujeres no son considerados asuntos de la misma importancia. Las críticas feministas a esta dicotomía y las propuestas elaboradas tanto desde el feminismo liberal (que aboga por una mayor participación femenina en la vida pública) como desde otras perspectivas feministas que defienden el derecho de las mujeres a decidir autónomamente sobre el propio cuerpo podrían trasladarse perfectamente a estos nuevos problemas que surgen en la red y afectan especialmente a las mujeres.

\section{Caso 3) Libertad y democracia en Internet}

Como apuntamos en la sección anterior cuando hablamos de la corriente ciberlibertaria en Internet, existe una autopercepción en estas comunidades de que conforman una nueva «cultura de la libertad» de corte meritocráitco, en la que todo el mundo puede participar sin discriminación de edad, raza, clase social, grado académico o profesional, etc $^{37}$. Es esta la típica expresión liberal acerca de la igualdad de oportunidades (lo que podríamos llamar «retórica de la igualdad») que, como ha sido criticado por el feminismo, no considera suficientemente que los diferentes colectivos no parten de la misma situación. La experiencia ha mostrado que la igualdad o la democracia no son propiedades que emergen espontáneamente de estas nuevas comunidades. Uno de los ejemplos más claros es que la participación de las mujeres en ellas es extremadamente baja $^{38}$. ¿Debe ser asunto de preocupación de la ética computacional la ausencia de mujeres de estas comunidades? Desde el feminismo es, precisamente, la principal preocupación. Es parte ya de la tradición feminista sobre tecnologías de la computación el rastreo por la historia de la informática en busca de «mujeres olvidadas» que han formado parte del desarrollo de esta disciplina. Tales son los conocidos casos de Ada Lovelace (creadora del concepto mismo de «lenguaje de programación lógico») o Grace Murray Hooper (inventora del lenguaje de programación COBOL). Asimismo podemos encontrar multitud de mujeres en las universidades y en las empresas que son expertas programadoras y tienen altos conocimientos de informática. Sin embargo estas mujeres no suelen participar de lo que se conoce como «comunidades hacker» que se caracterizan por ser comunidades informales «online» con el ideal de la autoorganización y la autonomía respecto de instituciones oficiales y empresariales.

Algunos de los primeros intentos de analizar el fenómeno hacker desde una perspectiva de género ${ }^{39}$ han partido del análisis del lenguaje sexista utilizado por las primeras comunidades hacker, y de la utilización de metáforas que se asocian 
fuertemente con la masculinidad como la de «frontera». Como han puesto de manifiesto los estudios sobre metáforas en la ciencia, y en concreto los estudios feministas sobre ciencia y tecnología, el uso de metáforas no es inocuo a la hora de dirigir en cierto modo el desarrollo de teorías científicas o desarrollos tecnológicos, y tampoco en el efecto de alejar a las mujeres de estos dominios. Alison Adam afirma que existe una fuerte asociación entre la tecnología en general con el deseo, el erotismo y el poder, lo que en el caso de la computación se lleva a su máxima potencia en el ámbito de la inteligencia artificial ${ }^{40}$. La cultura hacker recoge en su seno esta tradición, e incluye muy explícitamente el uso de la metáfora de la «frontera» en el sentido americano del «salvaje oeste». La idea de vivir en la frontera supone que las leyes y nociones de justicia que se aplican en la vida normal no operan debido a la naturaleza extraordinaria de la situación, de la que se deriva un excepcional grado de libertad. Los «exploradores» en este mundo fronterizo deben tomar la justicia por su cuenta, lo que les da un «estado de excepción» que, aunque puede ser peligroso, les confiere un alto grado de poder y libertad del que carecerían en circunstancias normales. Como señala Adam, es muy clara la relación tradicional entre los exploradores o pioneros y la celebración de su masculinidad (en términos de valentía, heroicidad y peligrosidad, tradicionalmente consideradas virtudes masculinas) ${ }^{41}$. En términos de género, por tanto, no es inocua la persistencia de esta metáfora en la imaginería popular en los textos sobre el ciberespacio y la cultura hacker. Esto tendría al menos dos consecuencias fácilmente analizables desde un punto de vista de género. Por un lado, la valorización positiva de este tipo de virtudes masculinas «de frontera» habría llevado a la minusvalorización o desestimación de contribuciones femeninas a la computación que no encajaran en esta ideología. Por otro lado, esta metáfora tendría como consecuencia el alejamiento del género femenino de este ámbito debido a que se perciben inevitablemente como «otras» dentro del mundo hacker (debido a que su socialización de género conlleva que sus «virtudes» y estereotipos no formen parte de este esquema). De este modo, sus aptitudes son relegadas en este caso al ámbito de «lo civilizado» 42 , es decir, fuera de la cultura hacker. Es fácil comprender, dado lo anterior, que a las mujeres les resulte muy difícil esquivar todos estos prejuicios y ejercer un rol diferente dentro de una comunidad que valora aptitudes que a ellas, por definición, les son negadas.

Esto es sólo un ejemplo de cómo puede ser aplicada la perspectiva de género en los acercamientos a la ética computacional y, en general, a los estudios sobre TICs y género, que, como hemos apuntado, puede tener infinitas variantes tal y como muestran los innumerables estudios feministas en el caso de la ciencia y la tecnología.

\section{Conclusión}

A lo largo de este artículo hemos intentado hacer un recorrido por los estudios sobre las nuevas tecnologías de la información y las comunicaciones, incidiendo en las ausencias de la perspectiva de género en los enfoques más asentados. Sin embargo, como hemos mostrado, existen ya algunos análisis de autoras feministas que están intentando incluir esta perspectiva. Por un lado, dentro de la corriente de CTS, los estudios feministas sobre tecnología cuentan ya con tres décadas de tradición, y en los últimos años parte de ellos se han dedicado a las tecnologías de la computación y la 
comunicación. Por otro lado, desde la tradición del feminismo cultural de corte postmoderno, el surgimiento del ciberfeminismo en los años 90 ha supuesto un fuerte impulso que ha proporcionado material de investigación para muchas autoras. En ambos casos, como hemos podido comprobar a lo largo de este artículo, los debates internos en el feminismo acerca de las tecnologías digitales han conducido a un gran desarrollo teórico que podría ser de mucha utilidad para comprender los problemas que actualmente plantean las TICs en su relación con la sociedad.

Un tercer ámbito de estudio del que nos hemos ocupado ha sido el de la ética computacional, mostrando cómo la perspectiva de género tiene muchas dificultades para ser incluida como parte constituyente de esta disciplina. El argumento principal que hemos sostenido es que esa ausencia dificulta la comprensión de los problemas éticos que suscitan las nuevas tecnologías computacionales en su total dimensión. Por tanto, no sólo reivindicamos la pertinencia de la perspectiva de género en la ética computacional por cuestiones de justicia (ya que la desigualdad de género afecta a la mitad de la población mundial), sino también por cuestiones «de contenido», es decir, porque creemos que la tecnología y el género se construyen mutuamente, por lo que obviar esta categoría de análisis conduce a una concepción sesgada y parcial del asunto en cuestión. Hemos querido mostrar esta tesis a través de tres ejemplos de problemas éticos que tienen que ver con las TICs en la sección anterior. A su vez, creemos que con una concepción parcial de un fenómeno, no es extraño que las propuestas éticas, políticas y legales que se apliquen para tratar de solucionar los problemas planteados tengan escasa o nula efectividad. Como muchas veces se ha argumentado desde los estudios CTS, si una disciplina (en este caso la ética computacional) no utiliza los recursos teóricos que ya se han producido en otros ámbitos (en este caso el feminismo) «reinventarán la rueda» una y otra vez.

¿Por qué creemos que el feminismo es tan importante para el estudio de las tecnologías y para la ética computacional? Principalmente porque la teoría crítica feminista parte de dos tradiciones diferentes, una de corte académico (con una importante fundamentación filosófica), y, por otro lado, una tradición activista que supuso uno de los movimientos sociales más importantes del siglo XX. Estas dos vertientes le dan al feminismo una situación inmejorable para una alianza entre teoría y práctica que no se ha dado aún en la mayoría de las disciplinas académicas, por lo que muchos de los problemas éticos y políticos planteados actualmente (entre ellos los relacionados con las tecnologías) se beneficiarían mucho de los debates largamente discutidos, y en parte solucionados, por la tradición feminista.

Lo que la ética feminista (a pesar de sus propios debates internos) puede ofrecerle a la ética computacional es un paradigma ético mucho más amplio que los esquemáticos parámetros que han tomado de las teorías éticas tradicionales, los cuáles han sido a su vez criticados por otras teorías éticas modernas. Por ejemplo, la reivindicación por parte de la ética feminista de un tipo de moralidad que incluya los valores del cuidado y la cooperación ${ }^{43}$, y valores como la responsabilidad medioambiental y la solidaridad ${ }^{44}$. Creemos que este tipo de valores son absolutamente necesarios para una resolución más justa de los problemas éticos que plantean las nuevas tecnologías TIC.

Tal y como ha criticado Langdom Winner ${ }^{45}$ a las tácitas asunciones de las comunidades ciberlibertarias y, en general, a la concepción tradicional sobre la 
tecnología, las nuevas tecnologías (en este caso Internet) no aseguran por sí mismas la justicia y la democracia, ni neutralizan las desigualdades, en este caso de género. Uno de los argumentos normalmente utilizados por estas posturas es el de que, debido a que se trata de una nueva tecnología que supone nuevas prácticas, no ha habido aún tiempo de que se establezcan los viejos estereotipos y comportamientos de género. Sin embargo, la experiencia nos ha mostrado que en cada nueva disciplina o desarrollo tecnológico vuelven a repetirse los mismos estereotipos. Winner asegura, incluso, que las nuevas relaciones a través de las TICs refuerzan y magnifican las asunciones tradicionales y los comportamientos discriminatorios, debido en parte a la facilidad y la posibilidad de anonimato que estas tecnologías proporcionan. Como ya mencionamos anteriormente, los ideales «ciberlibertarios» de democracia en Internet no se corresponden demasiado con la situación de desigualdad real en nuestras sociedades, ni con una participación equitativa de hombres y mujeres, ya que la libertad no funciona igualmente para aquellos cuya situación en la sociedad es de hecho discriminatoria.

Es necesario, en cambio, aplicar ideales morales (y prácticas concretas) de igualdad, responsabilidad, y solidaridad con las minorías y los grupos en posición desfavorecida, grupos que, a día de hoy, están ausentes en general de las comunidades virtuales en Internet. Sólo de este modo creemos que la ética computacional podría considerarse una disciplina ética en toda su dimensión.
* El trabajo para la elaboración de este artículo ha podido ser realizado gracias a la beca asociada al proyecto «Sociedad Civil y gobernanza de la ciencia y la tecnología en España», financiado por la FECYT y el CSIC. Quiero expresar mi agradecimiento por sus notas y comentarios a las profesoras Eulalia Pérez Sedeño y Marta González García, los cuales han sido de inestimable ayuda para la elaboración final de este trabajo.

${ }^{1}$ Por ejemplo Judy Wajcman, Susan Ormrod, Anne-Jorunn Berg, Merete Lie o Maria Lohan.

2 Véase Bijker, Wiebe E., Thomas P. Hughes and Trevor Pinch(eds), The Social Construction of Technological systems, The MIT Press, 1987

${ }^{3}$ Pinch, Trevor J. and Bijker, Wiebe E., «The Social Construction of Facts and Artifacts: Or How the Sociology of Science and the Socioligy of Technology Might Benefit Each Other», en Bijker et. al (Ibid) pp. 17-50.

4 Latour, Bruno, (1987) Science in Action, Buckingham, Open University Press. Traducción al español, Ciencia en Acción, Barcelona, Editorial Labor, 1992.

5 Op. Cit. Nota 3.

6 Siguiendo la teoría de la construcción del género de Judith Butler, éste se produce a través de un proceso preformativo de reiteración de actitudes, acciones y estereotipos.

${ }^{7}$ Grint, Keith and Rosalind Gill (eds). The Gender-Technology Relation: Contemporary Theory and Research. London: Taylor and Francis, 1995.

${ }^{8}$ Haraway, Donna, «Situated Knowledge: The Science Question in Feminism as a Site of Discourse on the Privilege of Partial Perspective.» In Simians, Cyborgs, and Women: The Reinvention of Nature. Routledge, 1991.

9 Wajcman, J., Technofeminism, Cambridge, UK: Polity Press, 2004, p. 7.

10 Haraway, D.: «A manifesto for cyborgs: science, technology and socialist feminism in the 1980's». Publicado inicialmente en Socialist Review, 80 (1985), y recogido como «A Cyborg Manifesto» en Simians, Cyborgs and Women: The Reinvention of Nature, London: Free Association Books, 1991.

11 Plant, S., Zeros and Ones: Digital Women and the New Technoculture, London: Fourth Estate, 1998. Traducción al español: Ceros + unos, Mujeres digitales + la nueva tecnocultura, Destino, Barcelona 1998.

12 El énfasis es nuestro, para mostrar nuestras dudas a cerca de la existencia de una «esencia femenina» universal. 
13 Turkle, S., Life on the Screen: Identity in the Age of the Internet, New Yor: Simon \& Shuster, 1995. Traducción al español: La vida en pantalla: la construcción de la identidad en la era de Internet, Paidós, Barcelona, 1997.

14 No obstante dentro de Ciberfeminsmo han surgido voces críticas respecto al excesivo optimismo de los primeros acercamientos y que mantienen otras posturas ciberfeministas, como por ejemplo Rosi Braidotti (1998), Cyberfeminism with a difference, en www.let.uu.nl/womens_studies/rosi/ cyberfem.htm, y, en España, Sonia Reverter-Bañón: «Ciberfeminsmo: entre la (u)topía y la (dis)topía» accesible en www.creatividadfeminista.org/articulos/ciber_utopia_disto.htm.

15 Para un recorrido completo de las diferentes corrientes en los estudios feministas sobre tecnología véase Sanz González V.: «Estudios Feministas sobre Tecnología. Un repaso desde los comienzos» en Clepsydra, 4, Enero 2005, pp. 97-112.

16 Castells M., The Rise of the Network Society, Oxford : Blackwell, 1996 y, en español, La Galaxia Internet, Areté, Barcelona, 2001.

17 Esta visión de Internet se fundamenta en la llamada «cultura hacker», muy apreciada por Castells, que estaría caracterizada por la cooperación, la reciprocidad y la comunicación horizontal a través de la red y cuyo principal valor es la libertad (de crear, distribuir y apropiarse del conocimiento disponible). Como veremos en la sección 3, la cultura hacker no es tan «ideal» en cuanto al valor de la equidad, concretamente de género.

18 Para una aproximación general a la materia véase la entrada «Computer Ethics» en la Stanford Enciclopedia of Philosophy, disponible en http://plato.stanford.edu/.

19 Un ejemplo de esta postura es Deborah Johnson en su libro Computer Ethics, Prentice Hall, 1985, que está traducido al español: Ética Informática, Universidad Complutense de Madrid, 1996.

20 Walter Manner, uno de los primeros académicos en usar el término «computer ethics» en los años 80 y distribuidor de materiales curriculares y pedagógicos acerca de esta materia, defiende esta postura en «Unique Ethical Problems in Information Technology», Science and Engineering Ethics, 2 (2), 1996 pp. 137-154.

21 La principal es la International Journal of Information Ethics (IJIE).

22 Uno de los primeros y más citados artículos es el de James Moor.: «What is computer ethics?» Metaphilosophy, 16 (4) 1985 pp. 266-75, y también los trabajos citados en las notas 16 y 17 .

23 Op. Cit. Nota 18.

24 Para una aproximación general a estas posturas véase Sterling, B.: The Hacker Crackdown:
Law and Disorder on the Electronic Frontier. London, Viking, 1992. En cuanto a sus demandas podemos consultar el ya famoso manifiesto de John Perry Barlow: «Declaración de independencia del Ciberespacio», 1996, disponible en www.arnal. es/free/info/manif-1.html y la llamada Carta Magna para la Edad del Conocimiento: Esther Dyson, George Gilder, George Keyworth, Alvin Toffler, "Cyberspace and the American Dream: A Magna Carta for the Knowledge Age,» Release 1.2, Progress and Freedom Foundation, Washington, D.C., August 22, 1994, en http://www.pff.org/issuespubs/futureinsights/fi1.2magnacarta.html.

25 Especialmente su último libro Gender, Ethics and Information Technology, Palgrave Macmillan, New York (NY), 2005.

26 Ejemplos de algunas de las primeras publicaciones de este tipo de estudios son: Lovegrove, G. and B. Segal. (1991): Women into Computing: Selected Papers 1988-1990, London \& Berlin, Springer-Verlag; Lander, R. and A. Adam, (1997): Women in Computing. Exeter, UK, Intellect; y en Estados Unidos por ejemplo Tracy Camp (1997): «The Incredible Shrinking Pipeline», Communications of the ACM, vol. 40, no.10, pp. 103-110.

27 La mayoría de este tipo de análisis se han desarrollado, como hemos apuntado, dentro de los estudios de ética empresarial, y suelen ser publicados en el Journal of Bussiness Ethics, por ejemplo: Khazanchi, D. : «Unethical behavoir in information systems: the gender factor» $1995, \mathrm{n}^{\circ} 15 \mathrm{pp}$. 741-749, o Mason, E. y P. Mudrack: «Gender and ethical orientation: a test of gender and occupational socialization theories» $1996, \mathrm{n}^{\circ} 16$, pp. 599 604. Una excepción a la perspectiva estrictamente empresarial es el artículo de Kreie, J. y T. Cronan: «How men and woman view ethics», publicado en Communications of the ACM, 1998, $\mathrm{n}^{\circ} 41$ (9): pp. 70-76.

28 Los datos son el primer caso para poner sobre el tapete la situación de desigualdad que la opinión general de la sociedad parece no creer «dados los tiempos en que estamos». En el Departamento de CTS del Instituto de Filosofía del CSIC analizamos el caso español: Pérez Sedeño, E. (2003): «La situación de las mujeres en el sistema educativo de ciencia y tecnología en España y su contexto internacional» Informe para el M.E.C., disponible en: http://wwwn.mec.es/univ/jsp/plantilla.jsp?id=2146. Por otra parte yo misma he realizado una investigación de este tipo en la Facultad de Informática de la Universidad Politécnica de Madrid que va a se presentada en la 6th Gender Research Conference en Lodz (Polonia) próximamente, y que será publicado en las actas con el título: «Women's careers in Computer Engineering. Case study: Technical University of Madrid» 
29 Véase Castaño, C, Las mujeres y las tecnologías de la información. Internet y la trama de nuestra vida, Madrid, Alianza Editorial, 2005.

30 Op. Cit Nota 22.

31 Op. Cit Nota 22.

32 Introducida por el psicólogo americano Rensis Likert en 1932, la escala que lleva su nombre es el instrumento más utilizado en psicología y ciencias sociales para medir las actitudes ante afirmaciones o juicios. Es una escala ordinal que se divide en cinco posibles respuestas (a veces pueden ser siete) del tipo: Muy de acuerdo, De acuerdo, Ni de acuerdo ni en desacuerdo, En desacuerdo y Muy en desacuerdo.

33 Muchas de estas explicaciones de la situación de desigualdad debido a diferencias de poder es fácilmente trasladable a los crímenes contra la infancia, pues claramente los niños y las niñas están en una situación de vulnerabilidad respecto de los adultos en un grado si cabe mayor.

34 Este ultimo caso nos recuerda la tradicional justificación del acoso femenino en términos de «ellas se lo han buscado», traslado en este caso a la red.

35 Véase por ejemplo Allen, A. L., Uneasy Acces: Privacy for Women in a Free Society. Totowa, NJ; Rowman and Littlefield, 1988.

36 Kramer, J. y C. Kramarae: «Gendered Ethics on the Internet» en Communication Ethics in an Age of Diversity, J.Makau y R. Arnett (eds) Urbana y Chicago, IL, University of Illinois Press, 1997 pp: 226-243.

37 Así es expuesto en uno de los primeros libros sobre la cultura hacker: Levy, S., Hackers. Heroes of the Computer Revolution, Harmondswoth, UK, Penguin, 1984. Nos resulta curioso cómo, en su descripción de las variables no discriminatorias, Levy no incluye explícitamente la no discriminación por cuestiones de género (ni tampoco por discapacidad), aunque, concediéndole el beneficio de la duda, podemos considerar que se incluyen en el «etcétera».
38 De hecho, de entre la mayoría de las esferas tecnológicas, las comunidades hacker son las que menos número de mujeres incluyen.

39 Por ejemplo Kramer y Kramarae, Op. Cit. Nota 29.

40 Véase Adam, A., Artificial Knowing; Gender and the Thinking Machine. London and New York, Routkedge, 1998.

${ }^{41}$ Por supuesto, muchas historiadoras feministas han criticado a su vez esta visión de la historia reciente norteamericana poniendo de manifiesto que los pioneros del oeste fueron tanto hombres como mujeres, aunque lo que se valoró como ideología fueron las virtudes «masculinas» del rudo vaquero, violento (y blanco), mientras que las mujeres eran representadas de un modo estereotipado (y a menudo no coincidente con la realidad) como vulnerables cuidadoras dedicadas a las labores cotidianas del hogar en la dura vida del oeste.

42 No así en otros casos donde lo femenino es relacionado con la naturaleza en oposición a la relación masculina con la cultura. Esto muestra la maleabilidad de la ideología de género a la hora de justificar la inferioridad del género femenino.

43 Debemos resaltar que nuestro punto de vista no es el considerar estos valores como exclusivamente femeninos (lo que supondría un esencialismo feminista), si no que, aunque relacionados y reivindicados tradicionalmente por las mujeres, son, y es deseable que así lo sean, valores universales.

$44 \mathrm{Si}$ bien es cierto que este tipo de éticas no son exclusivas del feminismo, no es menos cierto que todas las posturas éticas feministas incluyen este tipo de valores.

45 Winner, L. (1997): «Cyberlibertarian myths and the prospect for community», ACM Computers and Society 27 (3): pp. 14-19. Existe traducción al español: «Los mitos ciberlibertarios y sus prospectos para la comunidad», Revista digital de Educación y Nuevas Tecnologías, disponible en http:// contexto-educativo.com.ar/2000/2/nota-1.htm. 